\title{
Measurement of Small Variations in Optical Properties of Turbid Inclusions with Respect to Surrounding Turbid Medium
}

\author{
I. Bliznakova*, O. Vankov, T. Dreischuh, L. Avramov and D. Stoyanov \\ Institute of Electronics, Bulgarian Academy of Sciences, Sofia, Bulgaria
}

\begin{abstract}
Special optical system for non-invasive determination of small variations in the optical properties of homogeneous turbid inclusions embedded into large turbid medium is proposed and developed experimentally. Results for different choice of the optical parameters of both media are presented. The minimum detectable changes in the inclusion optical properties are estimated to be less than $5 \%$ with respect to the surrounding medium. It is shown that the output signals depend not only on the relative magnitude but also on the sign of the difference in optical properties of both media. The results could be used for developing techniques and algorithms for distinguishing of different kinds of abnormal formations.
\end{abstract}

PACS numbers: 87.15.N-, 87.64.-t, 87.64.Cc, 87.80.--y, 87.80.Ek

\section{Introduction}

Optical tomography methods that use near-infrared light $(750 \mathrm{~nm}<\lambda<900 \mathrm{~nm})$ to probe biological tissues [1] could be effectively used for the detection and characterization of different benign and malignant breast lesions due to the difference in their optical properties (e.g., absorption and scattering). The literature data display some differences in reduced scattering coefficients of normal and abnormal tissues (for carcinoma lesions they are slightly higher $\approx 10-25 \%$ than for the normal tissue; for cyst lesions they are lower - down to $10-50 \%$, for fibroadenoma lesions $\approx 30-80 \%$ higher than for the normal tissue) $[2,3]$. Thus, the development of reliable methods providing an opportunity to measure at least $5 \%$ difference in the reduced scattering coefficient of such small formations (as turbid inclusions) surrounded by normal tissues (turbid media) will allow their earlier detection and offer new opportunities for earlier intervention.

One of the basic problems of optical tomography technique is related to the low signal contrast in the optical range of different benign and malignant formations surrounded by a normal tissue. In this paper we present the experimental results from applying a method developed by us for determination of small variations in optical properties of small turbid inclusions embedded into a large homogeneous turbid medium.

\section{Experimental setup}

The experimental setup is shown in Fig. 1. A CW-laser diode emitting collimated sensing beam $(\lambda=850 \mathrm{~nm}$, $\left.P_{\text {out }}=27 \mathrm{~mW}\right)$ is used to illuminate a transparent $\mathrm{cu}-$ bic container of $2300 \mathrm{ml}$ volume filled with a liquid of defined concentration that simulates a turbid medium.

* corresponding author; e-mail: irbliznakova@abv.bg
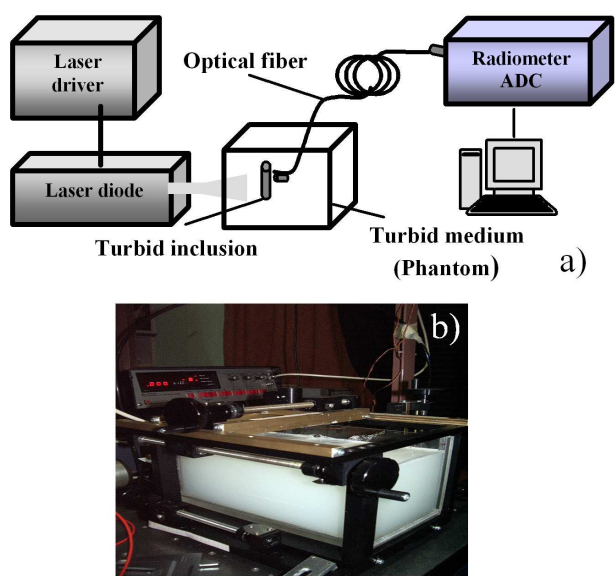

Fig. 1. Scheme of the experimental setup (a) and view of the developed mechanical system (b).

A cylindrical glass cuvette (10 ml volume) filled with a liquid with slightly different optical properties represents a small inclusion embedded into the medium. As a result of multiple scattering effects, the laser beam is spread within the turbid medium into a large spatial angle. The multiple scattered radiation is received by an optical fiber disposed within the phantom at a distance $2 \mathrm{~cm}$ from the inclusion and $6 \mathrm{~cm}$ from the front wall of the container (see Fig. 1a). Special mechanical system (Fig. 1b) allows perpendicular and parallel translation of the receiving optical fiber with respect to the beam axis. The collected radiation is measured by an optical radiometer (Laser Precision corp., USA) with RqP-546 silicon probe in external locking regime and the output signal is fed to the 14-bit analog-to-digital converter. The output digitized data are transferred to the computer and stored for further analysis (averaging, reducing the small optical power variations, etc.). 
As a phantom to simulate the optical properties of turbid tissue-like medium, a 20\% Intralipid (Fresenius Kabi) is used. Intralipid (IL) is an emulsion of soy bean oil and egg phospholipids and is widely used in optical experiments to simulate the scattering properties of biological tissues [4]. To obtain phantoms and inclusions with well defined but different optical parameters the concentration of the IL solutions is varied. Variations of the inclusions optical contrast are provided also by small amounts of blood added to the IL solutions [5]. In such arrangements (similar to some real measurement schematics) the useful signal, which depends on the difference of the optical properties of the medium and the small inclusion becomes very weak and requires high sensitivity and stability of the entire system.

\section{Experimental results and discussion}

The variations in the optical properties of the embedded small inclusions are measured by varying the concentration of the Intralipid solution up to $60 \%$ with respect to the surrounding medium. Five phantom concentrations are analyzed covering the typical turbidity of the breast tissues. As a first step, the scattered optical field in the absence of any inclusion in the phantom is measured. Then, measurements in the presence of inclusions with the same turbidity as the phantoms are performed (the only difference here is the influence of the glass cuvette). Finally, the measurements with increasing of IL and/or blood concentrations in the inclusions for various IL solutions of the phantoms are carried out.

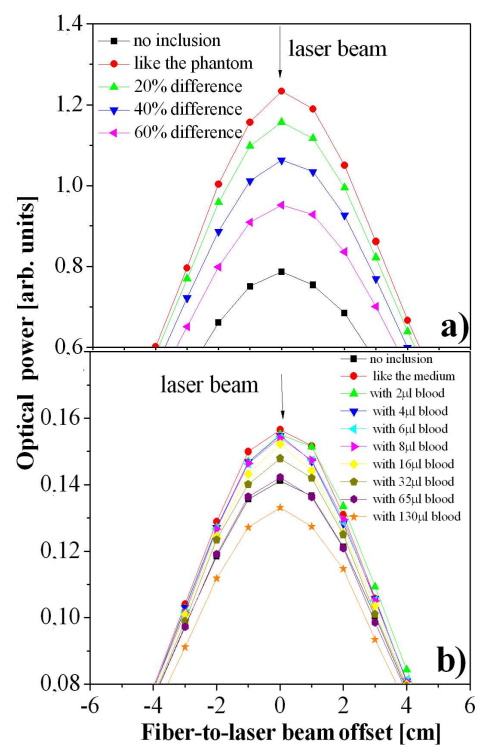

Fig. 2. Dependences of the experimentally measured signals in a transversal direction for different IL (a) and blood (b) inclusion concentrations for a phantom consisting of $60 \mathrm{ml} 20 \%$ IL emulsion dissolved in $2300 \mathrm{ml}$ water.

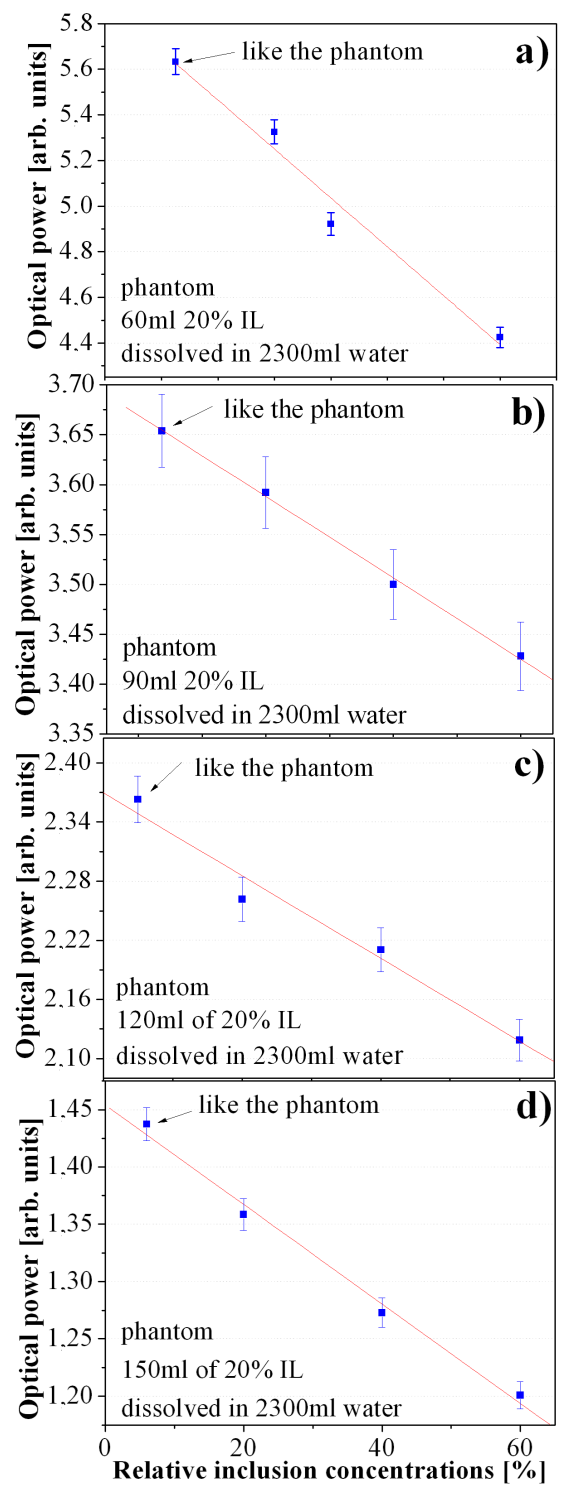

Fig. 3. Total scattered optical power as a function of the relative Intralipid concentration in the inclusion with respect to the phantom for different phantom concentrations.

The transverse distribution of the forward-scattered radiation intensity at fixed position of the laser and the inclusion (placed on the laser beam axis) is investigated by transverse scanning of the receiving optical fiber inside the medium. The dependences of the experimentally measured signals on the transverse receiver position for different IL concentrations of the inclusion are shown in Fig. 2a. The lowest curve corresponds to the absence of any inclusion in the phantom. The posing of an inclusion containing IL solution of the same concentration as in the phantom causes a strong increase of the signal because of the effect of the glass cuvette containing the inclusion. Then, the successive increase of the inclusion concentration with respect to the phantom concentration 
(with a step of $20 \%$ ) leads to a signal decrease as a result of increasing its turbidity. Thus, the method is sensitive to the sign of the difference in optical properties of both media - the glass cuvette (less scattering medium) causes an increase of the signal, while the increase of the inclusion concentration causes a decrease of the signal. As seen, there is a clear difference between the signals corresponding to $20 \%$ changes of the inclusion turbidity. The next Fig. 2b presents similar experimental measurements but for inclusions with a constant concentration of IL (equal to that in the phantom) and a varied concentration of blood. It is evident that the small changes in the added blood quantity are well detected by the measurement system.

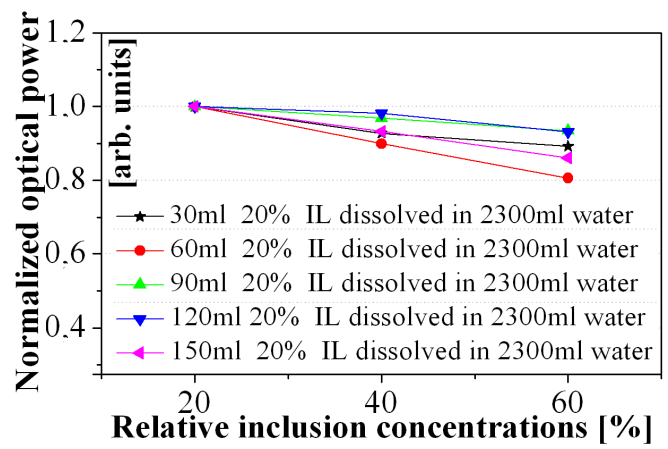

Fig. 4. Output power normalized to the lowest IL concentration in the inclusion for different phantom concentrations.

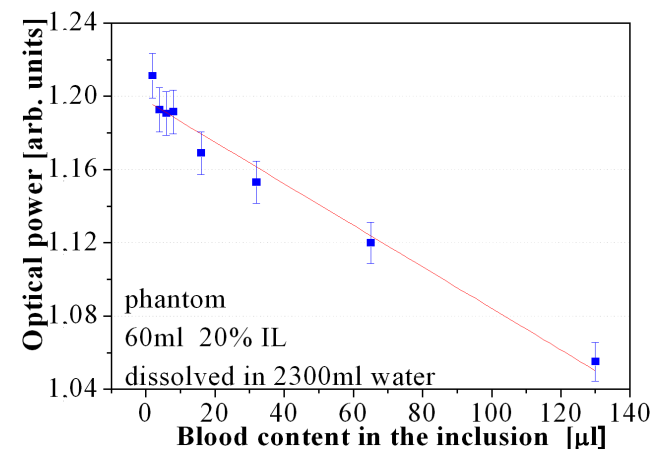

Fig. 5. Total scattered optical power as a function of the blood additions to an inclusion with a constant concentration of IL, equal to the phantom concentration (60 $\mathrm{ml} 20 \% \mathrm{IL}$ emulsion dissolved in $2300 \mathrm{ml}$ water).

Figure 3 illustrates the changes of the total scattered optical power (the areas of the curves shown in Fig. 2a) with increasing the relative IL concentration (from $20 \%$ to $60 \%$ ) in the inclusion with respect to the phantom. The results obtained for different phantom concentrations (from $60 \mathrm{ml}$ to $150 \mathrm{ml} 20 \%$ IL in $2300 \mathrm{ml}$ water) are presented. As seen, independently of the absolute phantom concentration, there is a linear dependence of the optical power on the relative inclusion concentration. In Fig. 4 we present the data from Fig. 3 normalized to the data for the lowest IL concentration in the inclusion. The variations in the slopes of the curves are probably due to some small errors in the measured concentrations or to some system instabilities. The similarity between the curves in Fig. 4 provides the opportunity to apply results obtained for a defined phantom to other media of different turbidity.

The total scattered optical power obtained in the measurements in the case of inclusions with a constant concentration of IL (equal to that in the phantom) and a varied concentration of blood (from $2 \mu \mathrm{l}$ to $130 \mu \mathrm{l}$, see Fig. 2b) is shown in Fig. 5. This figure illustrates the effect of small blood addition to the inclusion solution. As in Figs. 3 and 4, there is a linear dependence of the total scattered optical power on the blood content, similar to that for varied IL concentrations in the inclusion. As seen, the developed experimental setup provides a good detection of very small additions of the blood bellow $10 \mu \mathrm{l}$ in the cuvette of $10 \mathrm{ml}$ volume.

\section{Conclusions}

The presented results demonstrate the principal ability of the method developed to distinguish small relative changes (down to $5 \%$ ) in the scattering parameters of some spatial areas inside turbid media acting as turbid inclusions. Linear dependences of the output signal on the changes in the inclusion concentration as well as a similarity of linear dependences for wide range variations of the medium turbidity are shown. Moreover, the output signal is sensitive to the contrast variations of the inclusion scattering with respect to the medium. It is important to note that the method schematic is similar to some experimental practices assuming the posing of inclusions in their typical surrounding media. Thus, the method is a good candidate for development of techniques and algorithms for solving the so-called "specificity" problem in optical medical diagnosis. The method and experimental results offer also good opportunities for further development of equipment for laboratory analyses as well as for creation of some optical tomography medical instrumentation.

\section{Acknowledgments}

This work was supported by the National Science Fund of Bulgaria under grant DO-02-112/08 "National Center on Biomedical Photonics".

\section{References}

[1] A. Klose, U. Netz, J. Beuthan, A. Hielscher, J. Quant. Spectrosc. Radiat. Transfer 72, 691 (2002).

[2] R. Veen, H.J.C.M. Sterenborg, A.W.K.S. Marinelli, M. Menke-Pluymers, J. Biom. Opt. 9, (2004).

[3] P. Taroni, F. Arpaia, G. Danesini, R. Cubeddu, Phys. Med. Biol. 50, (2005).

[4] I. Driver, J.W. Feather, P.R. King, J.B. Dawson, Phys. Med. Biol. 34, 1927 (1989).

[5] T. McBride, Ph.D. Thesis, Dartmouth College 2001. 